\title{
Automated 4D analysis of dendritic spine morphology: applications to stimulus-induced spine remodeling and pharmacological rescue in a disease model
}

\author{
Sharon A Swanger ${ }^{1}$, Xiaodi Yao ${ }^{1}$, Christina Gross ${ }^{1}$ and Gary J Bassell ${ }^{1,2^{*}}$
}

\begin{abstract}
Uncovering the mechanisms that regulate dendritic spine morphology has been limited, in part, by the lack of efficient and unbiased methods for analyzing spines. Here, we describe an automated 3D spine morphometry method and its application to spine remodeling in live neurons and spine abnormalities in a disease model. We anticipate that this approach will advance studies of synapse structure and function in brain development, plasticity, and disease.
\end{abstract}

Keywords: dendritic spine morphology, fragile $X$ syndrome, automated image analysis, BDNF, dendritic spine remodeling, live cell imaging, 3D reconstruction, synapse, FMRP, PI3K inhibitor

\section{Background}

Dendritic spines are dynamic, actin-rich protrusions that form the postsynaptic compartment at most glutamatergic synapses [1]. Synapse strength is closely correlated with dendritic spine morphology, and synaptic activity regulates spine number and shape during brain development, behavioral learning, and aging [2-4]. In addition, abnormal spine morphology is prevalent in neurological diseases such as intellectual disabilities, autism spectrum disorders, schizophrenia, mood disorders, and Alzheimer's disease [5-7]. Although many details regarding the spine structure-synapse function relationship remain unclear, it is evident that spine morphology can impact excitatory neurotransmission and is an important aspect of neuronal development, plasticity, and disease [6,8-10].

The lack of automated methods for quantifying spine number and geometry has hindered analysis of the mechanisms linking spine structure to synapse function [11]. Cultured neurons are the primary model system for studying the basic mechanisms regulating neuronal structure and function as these mechanistic studies

\footnotetext{
* Correspondence: gary.bassell@emory.edu

'Department of Cell Biology, Emory University, 615 Michael St. NE, Atlanta, GA 30322, USA

Full list of author information is available at the end of the article
}

require complex designs and large sample sizes in order to produce meaningful results. While several recent reports have described automated algorithms for analyzing neuron morphology in vivo [12-18], few independent studies have validated these methods $[19,20]$ and there are no established methods for automated 3D spine analysis in cultured neurons. Son et al. developed an automated spine analysis algorithm using $2 \mathrm{D}$ images of cultured neurons, but 2D analyses do not consider a significant amount of information including all protrusions extending into the z-plane [21]. The majority of spine morphology studies have relied on manual measurements, which are time consuming, often biased by experimenter error and fatigue, and have limited reproducibility [14].

Here, we present, validate, and apply an automated 3D approach using the commercially available software program Filament Tracer (Imaris, Bitplane, Inc.). Filament Tracer has been used for automated spine detection in vivo, but geometric measurements were limited to spine head width [22,23]. Also, we have used Filament Tracer to facilitate spine density calculations in cultured neurons, but this analysis required manual validation and extensive editing of false-positive spines [24]. Now, our improved approach generates an accurate $3 \mathrm{D}$ 
reconstruction without any manual validation. Moreover, our approach can be applied to either fixed or live neurons as well as images acquired using either widefield fluorescence or confocal microscopy.

To demonstrate the applicability of our approach, we analyzed changes in spine morphology following acute brain-derived neurotrophic factor (BDNF) application in live hippocampal neurons. We verified our method by showing that acute BDNF treatment increased spine head volume, as was previously published [25]. Furthermore, we demonstrated that BDNF application induced rapid alterations in spine neck and length geometry and resulted in an overall maturation of the dendritic spine population within 60 minutes. We also applied our method to the study of aberrant spine morphology in a mouse model of fragile X syndrome (FXS), an inherited intellectual disability [26]. We not only accurately detected the established spine abnormalities in cultured neurons from this mouse model, but we also demonstrated that these abnormalities were rescued by inhibiting phosphoinositide-3 kinase activity, a potential therapeutic strategy for FXS [24]. These findings demonstrate that our approach is an efficient and accurate method for investigating dendritic spine development and plasticity as well as neurological disease mechanisms and therapies.

\section{Results and discussion}

\section{Automated detection and 3D measurement of dendritic spines}

The accurate study of dendritic spine morphology requires a method that incorporates effective neuron labeling with unbiased spine detection and measurement. To establish the most effective method for labeling and detecting spines in cultured hippocampal neurons, we tested several fluorescent markers including the lipophilic dye DiI and plasmids encoding soluble eGFP, membrane-tagged eGFP, and mRFPruby-tagged Lifeact, a small actin binding peptide [27]. The labeled neurons were fixed, and z-series images were acquired using a widefield fluorescence microscope. Following deconvolution, the images were analyzed with two different software programs: NeuronStudio, a program used for automated 3D neuron tracing in vivo [12], and Filament Tracer (Imaris, Bitplane, Inc.), a commercially available 3D tracing software. Universal parameters for accurate automated tracing of a large dataset could not be identified using NeuronStudio with any fluorescent label or using Filament Tracer with DiI-labeled or GFPexpressing neurons (data not shown). However, accurate 3D traces were automatically generated from images of Lifeact-ruby-expressing neurons (Figure 1a). While GFP is commonly used for morphological analyses, we found that generating accurate traces of GFP-expressing neurons required extensive manual editing of false-positive spines. Images of Lifeact-expressing neurons could be used to generate automated traces with universal parameters and no manual editing. Of note, Lifeactexpressing neurons have been previously shown to exhibit normal actin dynamics and dendritic spine morphology $[27,28]$. Consequently, we describe here the validation and application of an automated spine analysis method using Filament Tracer and images of Lifeactexpressing neurons.

To generate the 3D reconstructions for spine analysis, we selected a dendritic region that was $40-60 \mu \mathrm{m}$ in length and void of dendritic branch points and crossing neurites. A point within the dendrite and at the edge of the selected region was assigned as the dendrite starting point, and the following parameters were set: minimum dendrite end diameter $(0.75 \mu \mathrm{m}$; empirically determined to be the minimum dendritic width enabling accurate tracing), minimum spine end diameter $(0.215 \mu \mathrm{m} ; 2$ times the pixel width), and maximum spine length (5 $\mu \mathrm{m})[29]$. The dendritic segment was then traced and volume rendered using automatic thresholds without any additional manual input or editing. On occasion the algorithm inappropriately assigned dendritic protrusions as dendrites instead of spines, so we applied a mathematical filter that selected all dendritic protrusions $\leq 5 \mu \mathrm{m}$ in length and assigned them as spines. To validate the automated spine detection, spine density was calculated within the same dendritic regions using manual and automated analyses (Figure 1b). The automated measures accurately predicted the manual spine counts as determined by linear regression analysis (Figure 1c) [30]. The mean spine density (spines per $10 \mu \mathrm{m}$ ) did not significantly differ between the manual $(4.36 \pm 0.46)$ and automated $(4.47 \pm 0.41)$ analyses (Student's t-test, $P=$ 0.836 ), but there was a consistent trend toward higher spine density using the automated method. The coefficient of variation was lower for the automated results (0.9) as compared to the manual measurements (0.11), suggesting that automated spine detection was slightly more reproducible than manual detection.

While spine number reflects the quantity of excitatory synapses, spine geometry is linked with excitatory synapse function and is also an important outcome measure in dendritic spine studies [10]. Spine head size is positively correlated with postsynaptic density (PSD) size, cell surface GluA receptor number, and synaptic vesicle content in the associated presynaptic terminal [31,32]. Spine length and neck width likely affect calcium signaling within spines as well as signaling from the spine to the dendrite shaft [33-35]. To evaluate how effectively our approach measured spine geometry, automated measurements of spine head width, neck width, and length were compared to manual measurements 


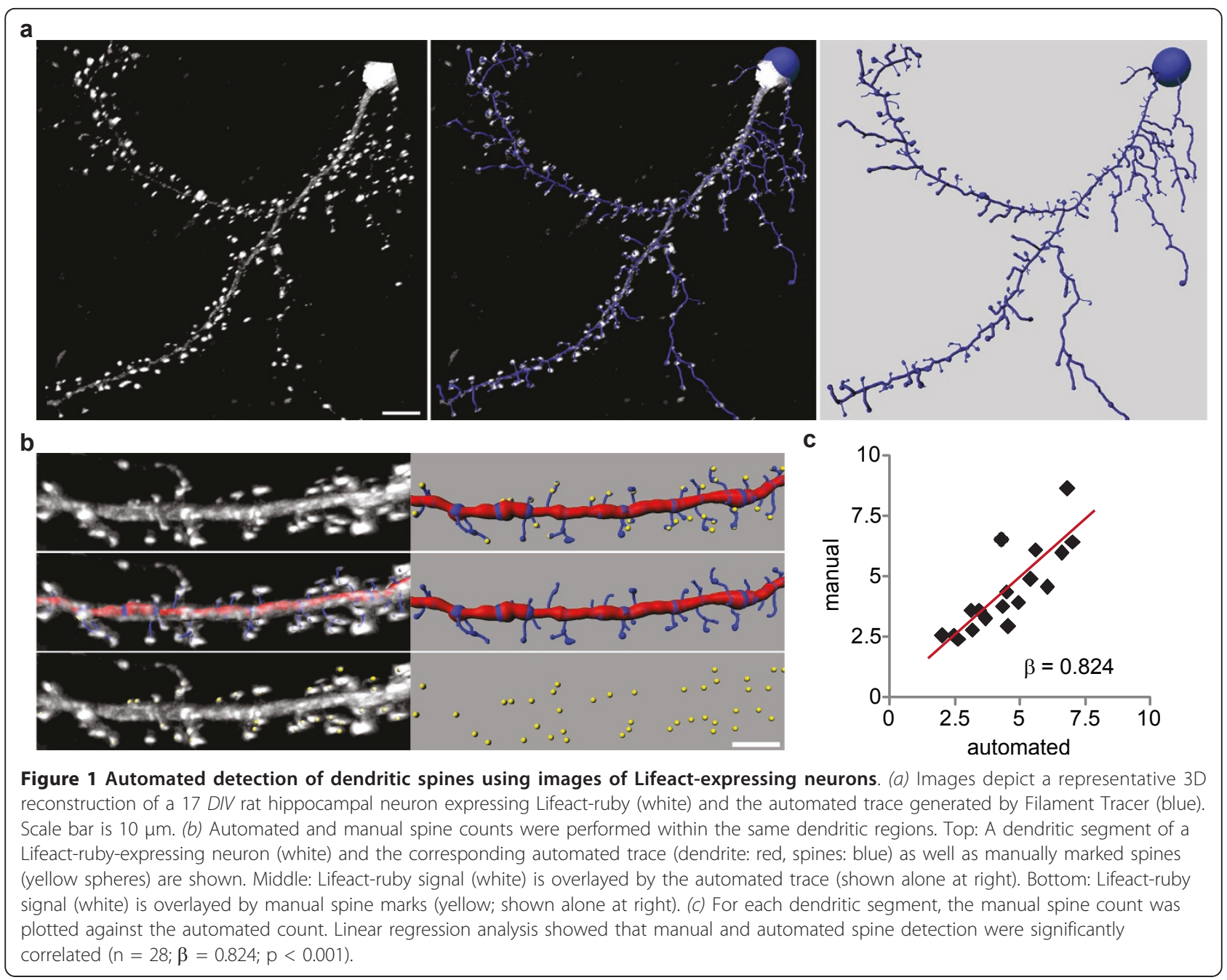

(Table 1). Unexpectedly, the distributions for each parameter significantly differed between the manual and automated methods $\left(N_{\text {manual }}=411 ; N_{\text {auto }}=423\right.$ spines; Kolmogorov-Smirnov Test; head width: $D=0.394$; neck width: $D=0.510$; length: $D=0.178$; all $P<0.001)$. Given these conflicting results, we evaluated the precision and accuracy of the automated and manual methods. To analyze precision, we evaluated specific characteristics of each dataset and found that the standard deviation and coefficient of variation were consistently smaller for the automated method (Table 1). Furthermore, the manual measurement distributions were more skewed than the automated distributions, indicating that the manual method yields distributions shifted further from the normal distribution as compared to automated analyses. Together, these data indicate that our automated approach is a more precise spine analysis method than manual measurements. To evaluate the accuracy of our approach, we used published ultrastructural data to estimate population statistics for spine head width, neck width, and length $[10,29,31,36]$. For each geometric parameter, the mean, median, and range values of the automated distributions (shown in Table 1) were more similar to the estimated population statistics (Table 2) than the manual values. For example, the estimated median head and neck widths garnered from several published ultrastructural studies were $0.40 \mu \mathrm{m}$ and $0.15 \mu \mathrm{m}$, respectively. Our automatically determined median head and neck widths were $0.46 \mu \mathrm{m}$ and $0.11 \mu \mathrm{m}$, respectively; whereas, our manually determined median head and neck widths were $0.60 \mu \mathrm{m}$ and $0.23 \mu \mathrm{m}$, respectively. These data suggest that the automated approach generated data that was more accurate than manual measurements.

In addition, dendritic spines were classified as stubby, mushroom, or thin using the aforementioned geometric measures; this is a widely used scheme to assess the proportions of mature and immature spines within a population [10,36,37]. While similar proportions of mushroom and thin spines were reported by both 
Table 1 Statistical comparison of geometric spine measurements

\begin{tabular}{lcccccc}
\hline & \multicolumn{2}{c}{ Head width } & \multicolumn{2}{c}{ Neck width } & \multicolumn{2}{c}{ Length } \\
\cline { 2 - 7 } & Manual & Auto & Manual & Auto & Manual & Auto \\
\hline Neurons & & & & & & \\
$\quad$ Mean $(\mu \mathrm{m})$ & 0.67 & 0.40 & 0.27 & 0.17 & 1.91 & 1.73 \\
$\quad$ SD & \pm 0.22 & \pm 0.08 & \pm 0.07 & \pm 0.03 & \pm 0.73 & \pm 0.26 \\
CV & 0.32 & 0.20 & 0.27 & 0.20 & 0.38 & 0.15 \\
Spines & & & & & & \\
$\quad$ Median $(\mu \mathrm{m})$ & 0.60 & 0.44 & 0.23 & 0.11 & 1.50 & 1.33 \\
$\quad$ Range $(\mu \mathrm{m})$ & 2.36 & 0.79 & 1.50 & 0.57 & 4.60 & 4.85 \\
$\quad$ Skewness & 1.25 & 0.10 & 2.66 & 2.09 & 1.03 & 0.84 \\
\hline
\end{tabular}

The mean, standard deviation (SD), and coefficient of variation (CV) were calculated for the manual and automated measurements of average spine head width, neck width, and length per neuron ( $\mu \mathrm{m} ; N=28$ neurons). The median, range, and skewness were calculated for the distributions of spine head width, neck width, and length determined using the manual ( $N=411$ spines) and automated methods ( $N=423$ spines) on the same 28 neurons.

methods, the manual method reported a significantly lower proportion of stubby spines than the automated method (Table 3). On close examination, we observed that spines classified as stubby by the automated method were often manually classified as thin, due to an increased length measurement, or were manually determined to be a region of the dendrite shaft rather than a protrusion. In agreement with these observations, it is evident from the literature that manual spine analyses consistently underestimate the proportion of stubby spines and overestimate spine length at the low end of the distribution when compared to automated and semi-automated methods $[12,17,38,39]$. It is important to note that, given the resolution limit of light microscopy, some spine heads may not be distinguishable from short and wide spine necks. While electron microscopy affords the resolution to make such distinctions, light microscopy is a more versatile and practical approach for mechanistic studies of dendritic spine structure. Altogether, these results indicate that our method accurately and precisely reports spine number and geometry in cultured neurons. Moreover, our method is a significant advance over current spine analysis methods as dendritic spine detection and 3D

Table 2 Estimated population statistics based on published electron microscopy studies

\begin{tabular}{lccc}
\hline & Head width & Neck width & Length \\
\hline Median & 0.40 & 0.15 & 1.36 \\
Mean & 0.46 & 0.15 & 1.50 \\
Range & 0.84 & 0.42 & 4.80 \\
\hline
\end{tabular}

Geometric spine measurements $(\mu \mathrm{m})$ from previously published electron microscopy studies were pooled to generate estimated median, mean, and range values for the population of dendritic spines on hippocampal neurons $[10,29,31,36,77]$.
Table 3 Statistical comparison of spine shape classification

\begin{tabular}{lcccccc}
\hline & \multicolumn{2}{c}{ Stubby } & \multicolumn{2}{c}{ Mushroom } & \multicolumn{2}{c}{ Thin } \\
\cline { 2 - 7 } & Manual & Auto & Manual & Auto & Manual & Auto \\
\hline Median & $7.0 \%$ & $13.5 \%$ & $65.5 \%$ & $59.4 \%$ & $11.7 \%$ & $9.3 \%$ \\
SD & \pm 11.4 & \pm 7.1 & \pm 20.0 & \pm 10.9 & \pm 15.5 & \pm 10.1 \\
CV & 1.10 & 0.49 & 0.33 & 0.18 & 0.99 & 0.80 \\
$P$ & \multicolumn{2}{c}{$0.033^{*}$} & \multicolumn{2}{c}{0.895} & \multicolumn{2}{c}{0.346} \\
\hline
\end{tabular}

Dendritic spines were classified as stubby, mushroom, or thin using the manually or automatically generated geometric measurements. $P$ : The Kolmogorov Smirnov test was used to compare the manual and automated distributions (*significant difference between the manual and automated measures)

measurements are entirely automated, thus greatly reducing the time burden and removing experimenter biases.

\section{Automated tracking of dendritic spines in live neurons}

Dendritic spine density and morphology are dynamically regulated by many extracellular cues and neurotransmitters. For example, many more dendritic protrusions are formed during development than remain into adulthood, indicating that spine formation and morphogenesis are highly regulated processes; yet, the mechanisms determining which spines become stabilized remain unclear. In the adult brain, stimulus induced potentiation of the postsynaptic response can convert spines with small heads to large spines, whereas large spines can shrink in response to long-term depression of the postsynaptic response $[40,41]$. However, the detailed mechanisms governing these differential responses remain poorly understood. Therefore, time-lapse imaging in living neurons is an essential tool for studying stimulus-induced synapse development and plasticity.

To test how effectively our automated approach tracked dendritic protrusions in live hippocampal neurons, $12 D I V$ neurons expressing Lifeact-ruby were imaged at $5 \mathrm{~min}$ intervals for $1 \mathrm{hr}$. The 3D reconstructions were generated as described above with a few modifications. A dendrite starting point was defined for each time point using the AutoDepth mode in Imaris Filament Tracer. The automated trace was built using these existing dendrite start points and the following geometric parameters: minimum dendrite end diameter $(0.75 \mu \mathrm{m})$, minimum spine end diameter $(0.3 \mu \mathrm{m}$; empirically determined to be the minimum end diameter allowing accurate spine detection), and maximum spine length $(15.0 \mu \mathrm{m})$. The maximum spine length was set at $15 \mu \mathrm{m}$ to include dendritic filopodia, which are long and dynamic protrusions involved in spine and dendrite development $[42,43]$. Filopodia are included in this analysis because they are abundant on the $12 \mathrm{DIV}$ neurons used for these experiments, whereas they are nearly absent on the mature neurons (17 DIV) used for 
the fixed neuron experiments described in Figure 1 and Table 1. The proportions of stable, new, and pruned dendritic protrusions (spines and filopodia) measured with the automated method were similar to those determined manually, suggesting that our automated approach allows detection and tracking of individual spines across time (Figure 2a). Moreover, we demonstrated that the morphology of individual spines can be tracked (Figure 2b) and quantified (Figure 2c and 2d) over time using our automated approach. Taken together, these data indicate that Lifeact-expressing neurons combined with this automated spine analysis approach is a valid method for the 4D tracking of dendritic protrusions in live neurons.

\section{Acute BDNF treatment induces synapse maturation through spine remodeling}

To test the usefulness of our approach, we analyzed the acute effects of BDNF on spine morphology in live neurons (Figure 3a). BDNF is a neurotrophin that not only supports neuron differentiation and survival, but it is also an important regulator of synaptic signaling and plasticity [44]. The canonical mechanism for BDNFinduced synapse maturation is through chronic exposure and a transcription-dependent pathway [45]. However, BDNF also enhances glutamatergic neurotransmission through rapid, local signaling events [44], and recently Tanaka et al. showed that acute BDNF treatment increased dendritic spine head volume by $\sim 150 \%$ within 25 minutes [25]. Here, we used cultured hippocampal neurons and our automated 4D approach to investigate the effects of acute BDNF application on dendritic spine morphology. Similar to the previous study, BDNF increased mean head volume by $\sim 160 \%$ within $20 \mathrm{~min}$, and this effect was maintained for $60 \mathrm{~min}$ (Figure 3a). In addition, we found that BDNF increased mean neck width by $125 \%$ (Figure $3 \mathrm{~b}$ ) and decreased mean protrusion length by $45 \%$ (Figure 3c). Spine classification analysis revealed significant increases in stubby and mushroom spine proportions and a decrease in the proportion of thin protrusions following BDNF treatment (Figure 3d). Finally, BDNF increased protrusion number by $\sim 25 \%$ (Figure $3 \mathrm{e}$ ). Together, these findings indicate that acute BDNF treatment leads to an overall maturation of the dendritic spine population in a manner consistent with enhanced synaptic efficacy.

In support of the above assertion, the observed increases in head and neck width and the decrease in protrusion length are associated with increased signaling between the dendritic spine and shaft, which promotes greater signal integration within the neuron [33-35]. Furthermore, we observed an increased proportion of mushroom-shaped spines, which have many GluA receptors and large PSDs; whereas, BDNF decreased the

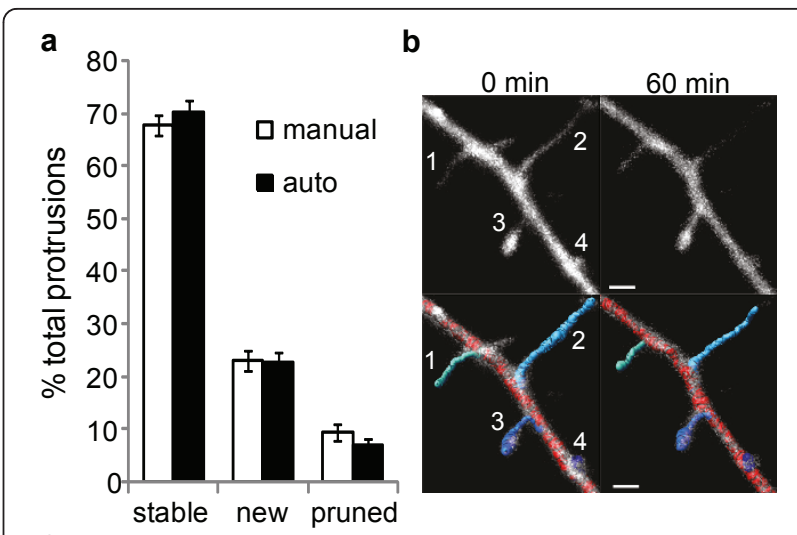

C

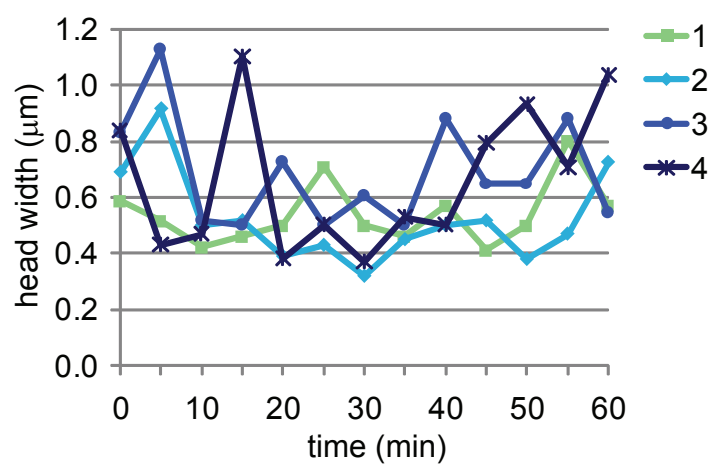

d

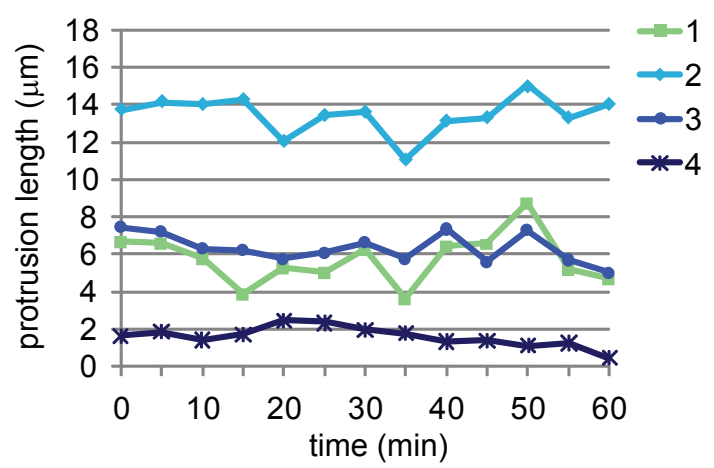

Figure 2 Automated tracking of dendritic protrusions in live neurons. 11 DIV hippocampal neurons were transfected with a vector expressing Lifeact-ruby, and 24 hrs later were imaged at 5 min intervals for $1 \mathrm{hr}$. (a) Individual protrusions were tracked across the time series manually and with our automated method. This histogram shows the percentages of stable, new, and pruned protrusions for both methods. (b) Images depict 12 DIV neurons expressing Lifeact-ruby (white; top) overlayed with automated 3D reconstructions (bottom). The dendrite shaft is red and each tracked protrusion is labeled 1 through 4 (shades of blue and green). Images from $\mathrm{t}=0$ and $60 \mathrm{~min}$ are shown, and the scale bar is 5 $\mu \mathrm{m}$. (c) Head width and (d) protrusion length were plotted versus time for each protrusion; the labels 1 through 4 in the legend correspond to the labels 1 through 4 in panel (b).

proportion of thin protrusions, which often lack surface GluA receptors and have less defined PSDs [46]. Importantly, our results agree with previous studies showing that acute BDNF enhances postsynaptic glutamate 


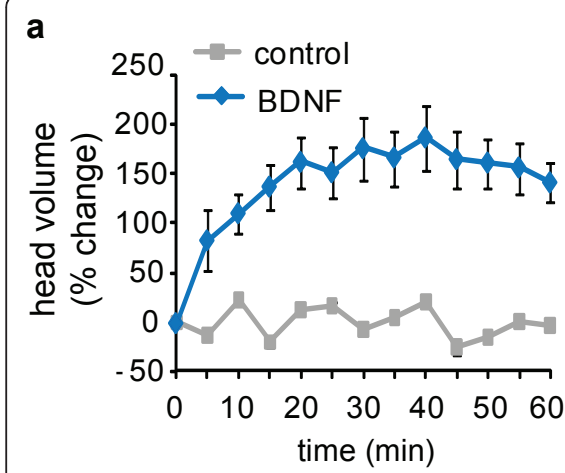

d

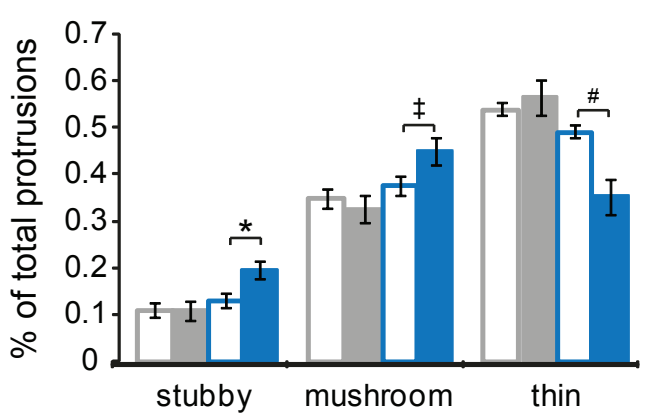

b

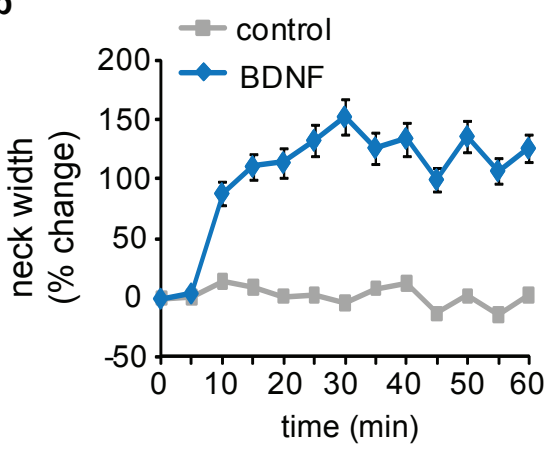

C

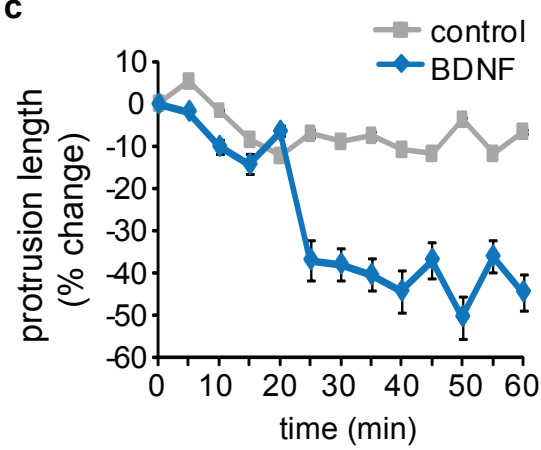

a control 0'

- control 60'

a BDNF 0'

BDNF 60'

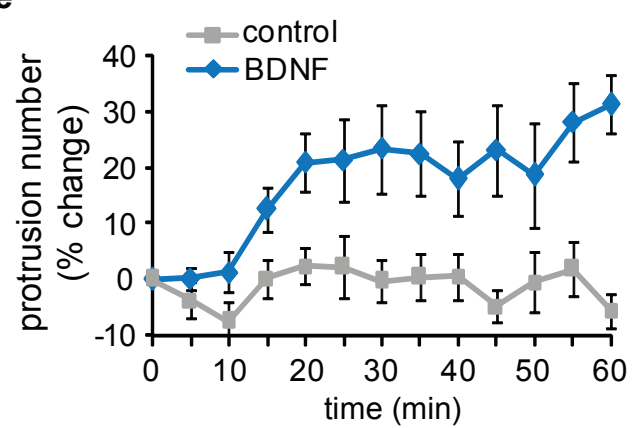

Figure 3 Acute BDNF treatment induces maturation of the dendritic spine population. 11 DIV hippocampal neurons were transfected with a vector expressing Lifeact-ruby, and 24 hrs later, the neurons were treated with vehicle or $100 \mathrm{ng} / \mathrm{ml}$ BDNF followed by time-lapse imaging every 5 minutes for $1 \mathrm{hr}$. Each protrusion was tracked across time and measured using the automated method. (a) Head volume was plotted as the percent change from the initial time point $\left(T_{0}\right)$. Statistical analyses were performed to compare protrusion head volume at $T_{0}$ and $T_{60}(N=$ $105-135$ spines; Kruskal Wallis test with repeated measures; Control: $P=0.548 ; \mathrm{BDNF}: P=0.001$ ). (b) Neck width was plotted and analyzed as above (Control: $P=0.91$; BDNF: $P=0.0002$ ). (c) Dendritic protrusion length was plotted and analyzed as above (Control: $P=0.648 ; B D N F: P=$ 0.017). (d) Dendritic protrusions were classified as stubby, mushroom, or thin at each time point. The percentages of total protrusions within each class are presented for $T_{0}$ and $T_{60}\left({ }^{*} P=0.038,{ }^{\ddagger} P=0.044,{ }^{\sharp} P=0.015\right)$. (e) The number of protrusions within a dendritic region was determined using Imaris Filament Tracer and compared between $T_{0}$ and $T_{60}(N=25-30$ neurons; repeated measures ANOVA, post-hoc Tukey's test [Control: $P=0.219 ; \mathrm{BDNF}: P=0.014]$ ).

receptor function, increases excitatory postsynaptic currents, and increases intracellular calcium concentration in hippocampal neurons [44]. Thus, our observations provide extensive morphological evidence supporting a role for BDNF in the acute regulation of synapse structure.

To determine how dendritic protrusions were remodeled to achieve the population effects described above, we tracked individual protrusions across time and quantified their morphogenesis. In this analysis, we asked three basic questions regarding remodeling: 1) does initial protrusion morphology affect remodeling, 2) what are the incidences of specific types of remodeling, and 3) what, if any, geometric parameters are associated with specific changes in morphology? We also evaluated whether BDNF treatment impacted these aspects of protrusion dynamics. Qualitatively, we observed several types of spine and filopodia remodeling such as: transient and highly dynamic thin protrusions, the morphogenesis of long, thin protrusions into mushroom-shaped spines, the growth of stubby-spines into mushroomshaped spines, and de novo mushroom spine formation (Figure 4a-d and sample movie in Additional File 1).

To quantitatively analyze remodeling, we calculated the percentages of each protrusion type (stubby, mushroom, or thin) that maintained classification, remodeled into another protrusion type, or were pruned over 60 min. All newly formed spines were excluded from this analysis. Under control conditions, similar proportions of thin protrusions and stubby spines were either remodeled into mushroom spines $(28.9 \%$ and $27.3 \%$, respectively) or pruned ( $26.3 \%$ and $18.2 \%$, respectively), whereas $78.6 \%$ of mushroom spines maintained their shape and only $7.1 \%$ were pruned (Figure $4 a$ ). Acute BDNF treatment increased the remodeling of both thin and stubby protrusions into mushroom spines $(40.5 \%$ and $42.9 \%$, respectively) as well as the percentage of mushroom spines $(26.7 \%)$ and thin protrusions (40.5\%) that were pruned. However, BDNF slightly decreased the percentage of stubby spines that were pruned 

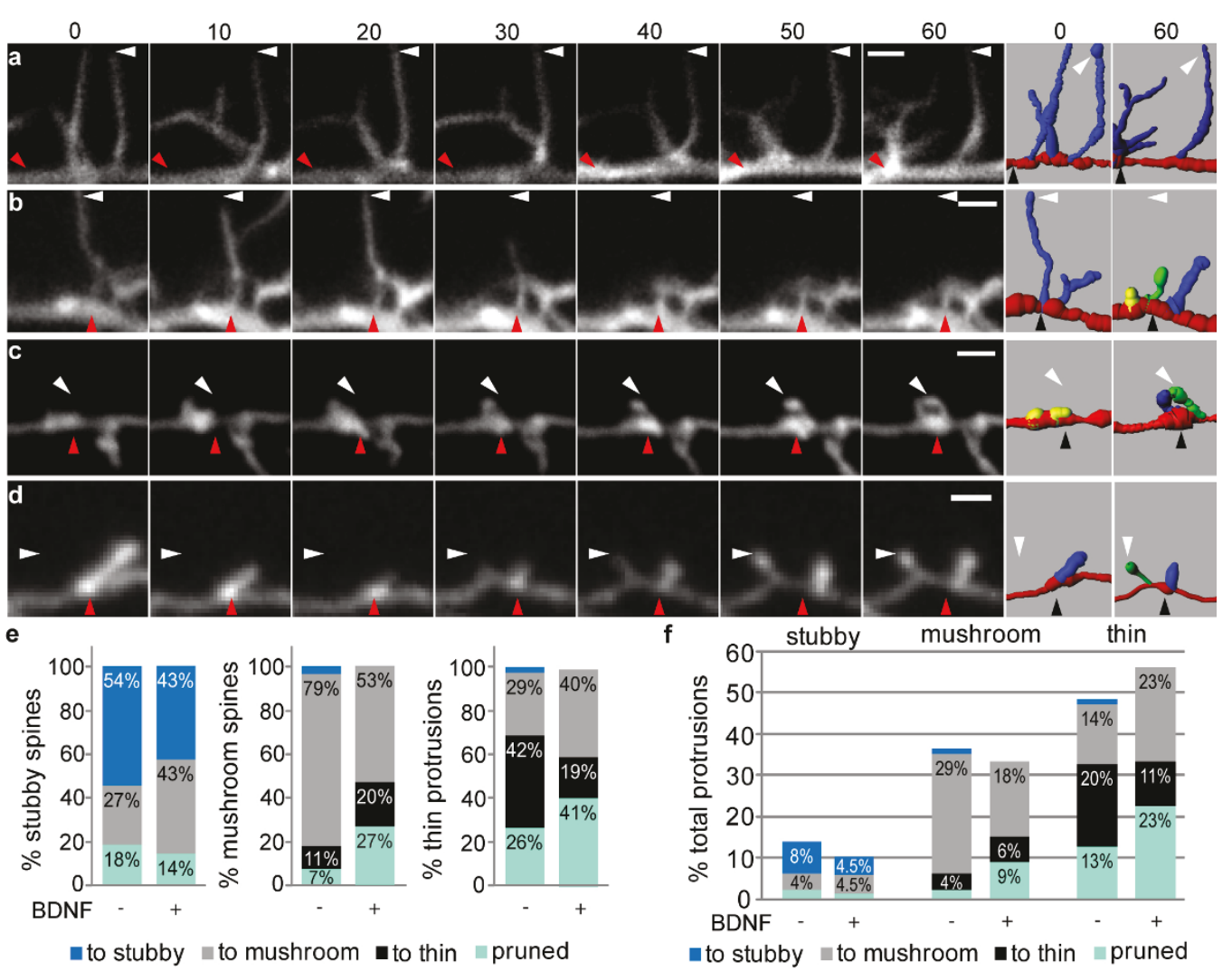

g

ato stubby $\backsim$ to mushroom $\square$ to thin $\square$ pruned

- to stubby $\backsim$ to mushroom $\square$ to thin $\backsim$ pruned

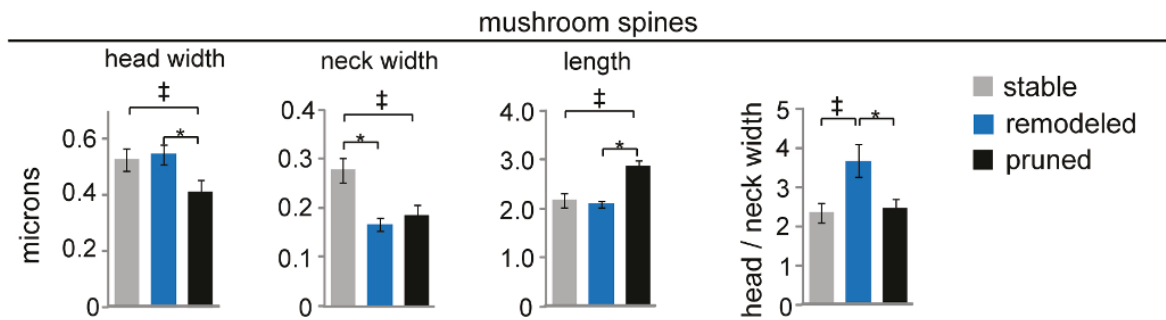

h

thin protrusions

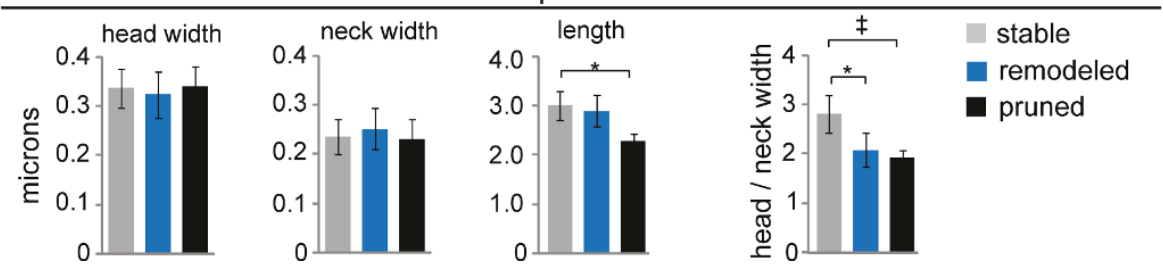

Figure 4 Acute BDNF induces specific types of spine remodeling. 12 DIV hippocampal neurons expressing Lifeact-ruby were treated with vehicle or $100 \mathrm{ng} / \mathrm{ml}$ BDNF and imaged at $5 \mathrm{~min}$ intervals for $1 \mathrm{hr}$. Each protrusion was classified as stubby, mushroom, or thin at $\mathrm{t}=0$ and 60 min. (a-d) At left, each each time series (0 - $60 \mathrm{~min}$ ) depicts a representative type of dendritic protrusion remodeling observed during our analysis. At right, the automated 3D reconstructions illustrate the classification of each protrusion at $\mathrm{t}=0$ and 60 min (stubby: yellow, mushroom: green, thin: blue). (e) The diagram shows the percentages of pre-existing stubby, mushroom, and thin protrusions that were remodeled (to stubby, to mushroom, to thin) or pruned under control and BDNF-treated conditions. (f) Using the same dataset as in (e), we calculated the total incidence for each type of remodeling under control and BDNF-treated conditions. The histogram depicts the percentages of total protrusions that were initially stubby, mushroom, or thin and were either remodeled (to stubby, to mushroom, or to thin) or pruned. ( $g$ ) The initial $(t=0)$ mean protrusion head width, neck width and length as well as the head width/neck width ratio were determined for mushrooms spines that were either stable (maintained mushroom morphology), remodeled into thin protrusions, or pruned within the 60 min imaging period following BDNF treatment ( $N=24$ - 32 spines; ANOVA, post-hoc Tukey's test; head width: ${ }^{*} P=0.032,{ }^{*} P=0.017$; neck width: ${ }^{*} P$ $=0.020,{ }^{\ddagger} P=0.037$; length: ${ }^{\ddagger} P=0.032,{ }^{*} P=0.017$; head/neck ratio: ${ }^{*} P=0.002,{ }^{\ddagger} P=0.001$ ). (h) The group means listed above were determined for thin protrusions $(t=0)$ that were stable (maintained thin morphology), remodeled into mushroom spines, or pruned during 30 min. BDNF treatment ( $N=36$ - 47 spines; ANOVA, post-hoc Tukey's test; length: ${ }^{*} P=0.002$; head/neck ratio: $\left.{ }^{*} P=0.037\right)$. 
(14.3\%). Interestingly, thin- and mushroom-shaped protrusions rarely morphed into stubby spines, and stubby spines were never observed to remodel into thin protrusions. These observations suggest that stubby and thin protrusions have similar propensities for remodeling into mushroom spines, but they likely do so through distinct mechanisms.

Among the total spine population, thin protrusions had the highest incidence of remodeling, and mushroom spines showed the lowest incidence of remodeling (Figure $4 \mathrm{~b}$ ). Following BDNF treatment, stubby spines had the lowest incidence of remodeling (see sample movie in Additional File 2), suggesting that stubby spines may not be simply a transitional structure, but that they might have an important end function as a stable structure under certain conditions. The BDNF-induced increases in the proportion and stability of stubby spines, reported in Figure 3, are difficult to interpret, because the role of stubby spines in neuronal function remains controversial. Stubby spines do not maintain or recruit GluA receptors as efficiently as mushroom spines, nor do they form synapses as often [47-50]. On the other hand, stubby spines might have enhanced coupling to the dendritic shaft as compared to the other spine types [51]. Also, stubby spine incidence is increased during learning in vivo, and it has been theorized that they are transitional structures that will be enlarged/stabilized or have undergone shrinkage due to synaptic weakening $[2,6,41,46,52,53]$. Our data suggest that it is unlikely for an increase in stubby spines to result from the weakening of mushroom spines or the retraction of thin protrusions, but it is possible that the increase in stubby spines is linked to the increase in total protrusion number following BDNF stimulation. Future studies in systems having a higher overall incidence of stubby spines, perhaps neurons in an earlier developmental stage, will be important for advancing our understanding stubby spine formation, remodeling, and function.

These results also have implications regarding spine formation. Several mechanisms have been proposed for how stable, mushroom-shaped spines are formed, including growth of mushroom spines from the dendritic shaft, morphogenesis of a filopodia into a mushroom spine, and retraction of filopodia into the dendritic shaft resulting in a shaft or stubby spine synapse followed by growth of a mushroom spine at the same location [47,54-56]. Our data clearly support the formation of mushroom spines de novo and through morphogenesis of an existing filopodia (Figure 4) as has been previously observed in vitro and in vivo [53,55-63]. However, our data suggest that mushroom spine formation via filopodia retraction into a stubby spine followed by re-growth is not a common occurrence, at least in this model system, as we rarely observed morphogenesis of a filopodia into a stubby spine. Whether filopodia were retracted fully into the shaft and re-emerged as mushroom spines at the same locus was not evaluated in the current study, but this analysis is possible using our automated method and can be investigated in future studies.

To investigate whether any geometric parameters were associated with BDNF-induced remodeling, the initial ( $t$ $=0$ ) mean head width, neck width, and protrusion length were compared among stable, remodeled, and pruned mushroom spines or stable, remodeled, and pruned thin protrusions (Figure 4g,h). Large neck width was the best predictor of mushroom spine stability, whereas head width was not significantly different between stable and remodeled mushroom spines (Figure $4 \mathrm{~g}$ ). Mushroom spine pruning was associated with reduced head and neck width and increased length compared to the other two groups (Figure $4 \mathrm{~g}$ ). For thin protrusions, a high ratio of head width to neck width was the best indicator of stability (see Figure $4 \mathrm{~d}$ and the sample movie in Additional File 3). Interestingly, these data are consistent with functional studies reporting that large neck width is associated with greater synaptic strength [33] and synaptic potentiation of thin protrusions is promoted by maintaining high concentrations of signaling molecules within the head, which might be due to a high ratio of head width to neck width $[46,64]$.

An interesting observation was that BDNF decreased the percentage of mushroom spines that remained as mushroom spines from $79 \%$ to $53 \%$ (Figure 4e). Moreover, $26 \%$ of mushroom spines were pruned following BDNF. Both observations imply BDNF-induced turnover of mushroom spines, suggesting that the overall net gain in spine maturation (increased density and spine width, reduced length) (Figure 3) involves extensive remodeling. This process may involve pruning of mushroom spines that passed certain thresholds approaching immature phenotypes (e.g. low head or neck width, or increased length), which are apparently replaced by more mature mushroom spines developed from other less mature populations (thin, stubby).

In the future, it will be important to study the different mechanisms underlying specific types of spine formation and remodeling, such as the distinctions between stubby and thin protrusion remodeling into mushroom spines. Furthermore, there is still much debate regarding the functional significance of different spine morphologies in brain development, plasticity, and disease. One necessary step towards understanding the structure-function relationship of dendritic spines is generating reproducible and interpretable spine morphology data. The accuracy and speed of our method makes it well-suited for studies of this type, and we 
anticipate that our approach will facilitate studies on spine structure and its relation to synapse function.

In addition to advancing morphological studies, the described technique has the potential to facilitate studies evaluating the synaptic localization of specific molecules. The fluorescence intensity of multiple channels can be automatically quantified within each dendritic spine; thus, one could evaluate whether a particular fluorescently tagged or stained molecule is differentially localized between spine types or shows altered localization following a pharmacological, molecular, or genetic manipulation. Therefore, the combination our optimized spine analysis method with automated quantification of spine fluorescence creates a powerful and efficient technique for simultaneously studying spine morphology and the molecules regulating synapse structure and function.

\section{Inhibiting PI3 kinase activity rescues dendritic spine defects in neurons from Fmr1 KO mice}

The importance of dendritic spine morphology is emphasized by the fact that spine abnormalities are associated with varied neurological diseases such as intellectual disabilities, neurodegenerative diseases, and psychiatric disorders [5]. Cultured neurons are a valuable model system for studying the mechanisms underlying brain diseases; as such, it is critical that spine analysis methods effectively detect aberrant spine phenotypes in disease models and identify treatments that ameliorate disease phenotypes. Here, we used our approach to study spine morphology in neurons from Fmr1 knockout mice, a mouse model of fragile X syndrome (FXS).

FXS is an inherited intellectual disability caused by the loss of fragile $\mathrm{X}$ mental retardation protein (FMRP), an RNA binding protein that regulates mRNA transport and local protein synthesis at synapses [26]. Patients with FXS display increased dendritic spine density, an increased incidence of thin spines, and increased mean spine length, and these abnormalities are recapitulated in neurons from Fmr1 knockout mice [65-68]. In a previous study, we detected increased spine density in cultured hippocampal neurons from Fmr1 knockout mice using a semi-automated spine analysis method in which the experimenter manually edited an automated trace; however, we were unable to detect any other defects in spine morphology with this method [[27], and unpublished observations]. Using our fully automated method, we accurately detected the established spine phenotypes in 18 DIV hippocampal neurons from Fmr1 knockout mice: increased spine density, decreased spine head width, increased spine length, and decreased spine volume (Figure 5). Furthermore, there were less mushroom-shaped spines and more thin spines in FMRP- deficient neurons, which is in line with previous reports [66,69-71]. These data further demonstrate the validity of our approach as well as its usefulness for studying neurological diseases.

Next, we investigated whether treating hippocampal neurons with a phosphoinositide-3-kinase (PI3K) inhibitor affected spine morphology. Previously, we discovered that inhibiting PI3K activity is a potential therapeutic strategy for FXS. We showed that the loss of FMRP leads to excess PI3K activity and treatment with a PI3K inhibitor, LY294002, rescues several neuronal phenotypes in Fmr1 knockout mice, including aberrant synaptic protein synthesis, GluA receptor internalization, and dendritic spine density [24]. Here, using our automated approach, we reproduced our previous findings by demonstrating that LY294002 treatment $(10 \mu \mathrm{M}$ for 72 hrs) reduced spine density in hippocampal neurons from Fmr1 knockout mice to wild type levels (Figure 5b). Furthermore, our analysis revealed that LY294002 treatment significantly increased spine head width, decreased spine length, and increased spine volume in neurons from Fmr1 KO mice (Figure 5c-e). Additionally, LY294002 significantly increased mushroom-shaped spines and decreased thin spines in FMRP-deficient neurons such that all spine proportions were similar to those of wild type neurons (Figure 5f). These data indicate that inhibiting PI3K activity not only rescues increased spine density in a mouse model of FXS, but also restores aberrant spine shape to the wild type morphology. These findings are an important advance of our previous findings and further support the pharmacological inhibition of PI3K as a potential FXS treatment strategy [72]. More broadly, these data demonstrate that our automated approach can be used to study dendritic spine abnormalities and potential pharmacotherapeutics in neurological disorders.

Although spine defects are apparent in many brain diseases, a vital unanswered question is whether altered spine morphology contributes to disease onset and progression or is secondary to disordered neuronal activity $[5,73]$. Of note, cortical neurons in a mouse model of Alzheimer's disease exhibit reduced spine density, a phenotype evident in patients with Alzheimer's disease, but these neurons do not show overt electrophysiological impairments; whereas, other mouse models of Alzheimer's disease show both structural and functional phenotypes in cortical neurons [74]. In addition, it is possible to alter synaptic efficacy without inducing longterm changes in spine morphology, and altering spine morphology through manipulating the neuronal cytoskeleton is not always sufficient to alter synapse function [4]. These data highlight the complexity inherent in the spine structure-synapse function relationship and emphasize the importance of developing powerful 


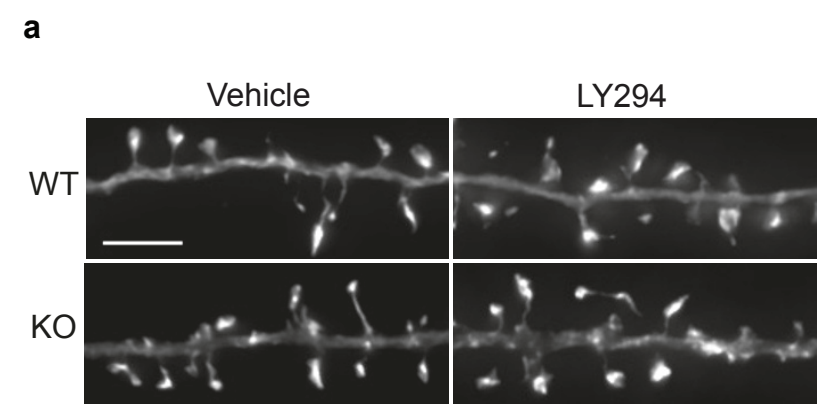

b

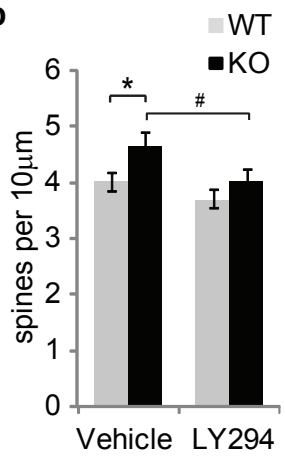

C

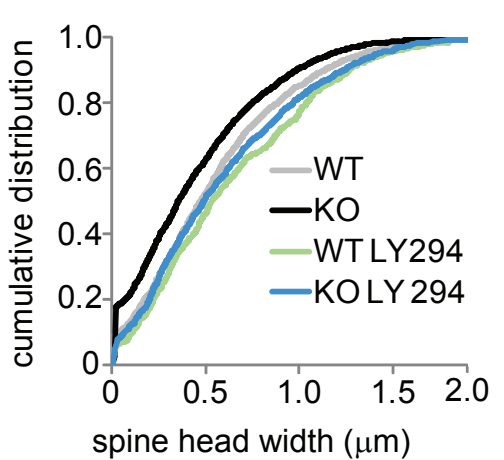

d

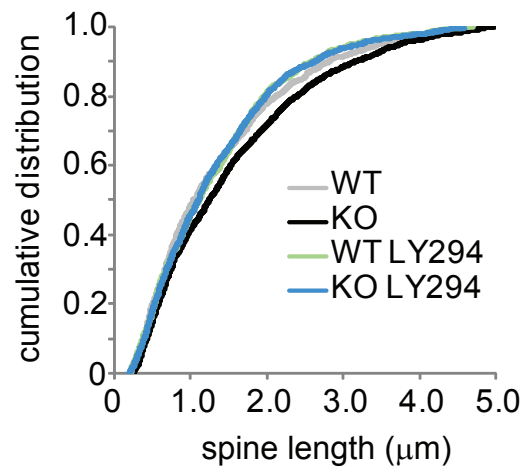

e

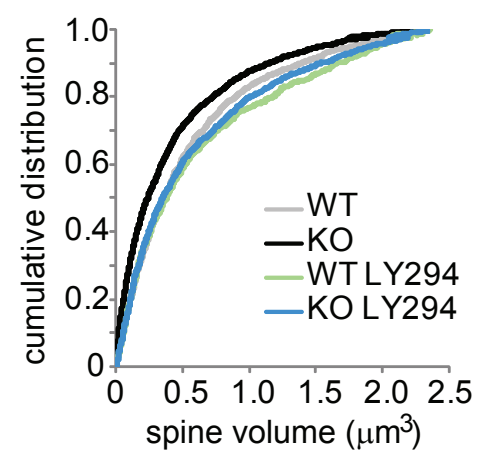

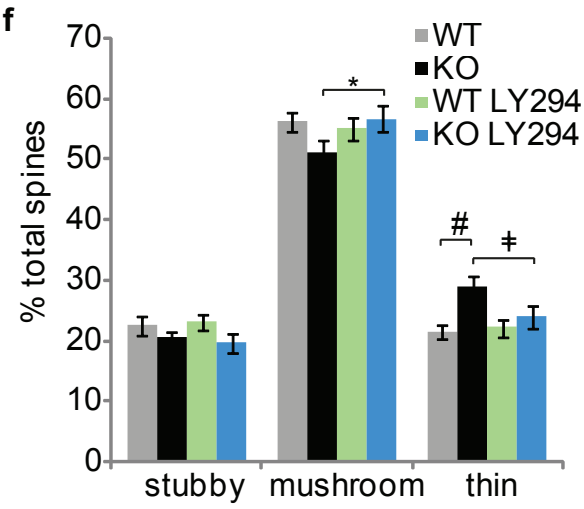

Figure 5 A PI3 kinase inhibitor rescues spine morphology in neurons from Fmr1 knockout mice. (a) Hippocampal neurons cultured from wild type (WT) or Fmr1 knockout (KO) mice were treated with vehicle or a PI3 kinase inhibitor (LY294, $10 \mu M)$ for 72 hours starting at 15 DIV. Neurons were transfected with a plasmid encoding Lifeact-ruby at 17 DIV and fixed 24 hours later. The images depict representative dendritic regions from deconvolved $z$-series images (scale bar is $5 \mu \mathrm{m}$ ). (b) Spine density was measured using our automated approach for WT and KO neurons treated with either vehicle or LY294 ( $n=55-60$ neurons; ANOVA $[F=3.996, P=0.009]$; post-hoc Fisher's LSD: ${ }^{*} P=0.017,{ }^{\#} P=0.028$ ). Cumulative distributions of (c) spine head width, (d) length, and (e) volume were plotted for each group (Kolmogorov-Smirnov test: head width [WT vs. KO: $P=0.002$, WT vs. WT LY294: $P=0.235$, KO vs. KO LY294: $P=0.009$ ], spine length [WT vs. KO: $P=0.009$, WT vs. WT LY294: $P=0.537$, KO vs. KO LY294: $P=0.014$ ], spine volume [WT vs. KO: $P<0.001$, WT vs. WT LY294: $P=0.158$, KO vs. KO LY294: $P<0.001])$. (f) Spines were classified as stubby, mushroom, and thin based on the automated geometric measurement, and the values were plotted as percentage of total spines per treatment group ( $\mathrm{n}=55$ - 60 neurons; ANOVA with post-hoc Fisher's LSD: ${ }^{*} P=0.043, \# P=0.006,{ }^{\ddagger} P=0.043$ ).

techniques for studying the mechanisms regulating spine morphology in brain development, plasticity, and disease.

\section{Conclusions}

We have developed an automated 3D approach for dendritic spine analysis using neurons expressing fluorescently labeled Lifeact. This versatile method can be applied to images of either fixed or live cultured neurons that were collected using widefield fluorescence or confocal microscopy. The increased speed and accuracy of our automated spine analysis, as compared to manual spine assessments, is critical for uncovering the complicated mechanisms underlying normal and aberrant dendritic spine formation 
and remodeling. Using our automated approach, we showed that acute BDNF treatment leads to rapid spine remodeling consistent with enhanced synaptic efficacy. We also found that inhibiting PI3 kinase activity rescues aberrant spine shape in neurons from a mouse model of FXS. We predict that this method will significantly advance studies of glutamatergic synapse structure and function in neuronal health and disease.

\section{Methods}

\section{Neuron culture, transfection, and drug treatments}

Hippocampal neurons were isolated from embryos at E18 (rat) or E17 (mouse) and cultured at high-density as previously described with minor modifications [75]. Rat hippocampal neurons were cultured in Neurobasal medium (Invitrogen) supplemented with NS21 [76]. Neurons were either plated on $15 \mathrm{~mm}$ glass coverslips and cocultured with glia, or plated on $35 \mathrm{~mm}$ MatTek glass bottom dishes in glia-conditioned media that was exchanged every 2 days with new glia-conditioned media.

Fixed neuron experiments: 16-17 DIV neurons were transfected with plasmids encoding Lifeact-ruby (a generous gift from Dr. Roland Wedlich-Soldner, Max Planck Institute, Martinsried, Germany), Lifeact-GFP, GFP, or membrane-tagged GFP using NeuroMag (OZBiosciences). DiI labeling was performed on 16 DIV neurons by incubating the coverslips covered with a small volume of neuronal culture media containing Vybrant DiI solution (Invitrogen) for $25 \mathrm{~min}$ at $37^{\circ} \mathrm{C}$. For LY294002 experiments, 15 DIV neurons were treated with $10 \mu \mathrm{M}$ LY294002 or an equivalent volume of DMSO for 72 hrs total; the culture media was exchanged with conditioned media containing freshly prepared drug (or vehicle) after 24 and 48 hrs.

Live neuron experiments: $11 \mathrm{DIV}$ rat hippocampal neurons were transfected with a plasmid encoding Lifeact-ruby using Lipofectamine 2000 and used for imaging $24 \mathrm{hrs}$ later. Thirty minutes prior to imaging, neurons were equilibrated to glia-conditioned imaging media (phenol red-free Neurobasal media supplemented with HEPES, sodium pyruvate, NS21, and Glutamax). For BDNF experiments: One hour prior to imaging, neurons were starved in glia-conditioned imaging media without NS21, and immediately prior to time lapse imaging neurons were treated with BDNF (100 ng/ml; Peprotech) or vehicle $\left(\mathrm{H}_{2} \mathrm{O}\right)$.

\section{Microscopy}

Widefield fluorescence: Twenty-four hours after transfection, hippocampal neurons were fixed with $4 \%$ paraformaldehyde in $1 \mathrm{x}$ phosphate-buffered saline (PBS), washed 3 times with $1 \mathrm{x}$ PBS, and the coverslips were mounted on microscope slides with propyl gallate- containing polyvinyl alcohol. Neurons were imaged on a Nikon Eclipse Ti microscope with a Nikon Intensilight and Photometrics Coolsnap HQ2 camera. GFP was imaged using a 480/40 excitation filter, a 535/50 emission filter, and a 505 dichroic (Nikon), and ruby and DiI were imaged using a 545/30 excitation filter, a 620/60 emission filter, and a 570 dichroic. Images were acquired using a $60 \mathrm{X}$ oil-immersion objective (Nikon Plan Apo, N.A. 1.40). Z-series images were acquired at $0.15 \mu \mathrm{m}$ increments through the entire visible dendrite.

Confocal laser scanning: Time lapse imaging was performed on a Nikon A1R confocal encased in a plexiglass humidified chamber maintained at $37^{\circ} \mathrm{C}$ and $5 \% \mathrm{CO}_{2}$ using a $60 \mathrm{X}$ oil immersion objective (Nikon Plan Apo, N.A. 1.40). Images of Lifeact-ruby were collected using a $561 \mathrm{~nm}$ laser for excitation and a 650 emission filter. Zseries were acquired at $0.15 \mu \mathrm{m}$ increments, and a Nikon Perfect Focus system was enabled for the duration of the experiment.

\section{Image processing}

Images were deconvolved in AutoQuant X (MediaCybernetics) using the blind algorithm, which employs an iteratively refined theoretical PSF. No further processing was performed prior to image analysis. For preparation of figures, maximum intensity Z-projections were created in Imaris (Figures 1 and 5) or average intensity Zprojections were created using ImageJ (Figures 2 and 4). For visualization, brightness and contrast levels were adjusted using ImageJ.

\section{Automated image analysis}

In Imaris Surpass mode, a new filament was created using the Autopath mode and a region of interest (ROI) was selected. To select an ROI, we identified a dendritic region 40 - $60 \mu \mathrm{m}$ length that was distal to a dendritic branch point and void of crossing neurites or any additional dendritic branch points. A minimum dendrite end diameter of $0.75 \mu \mathrm{m}$ was entered and a single dendrite starting point was assigned at the edge of the ROI. For time-lapse image series, a single dendrite starting point was assigned at each timepoint by using the AutoDepth mode. Automatic thresholds were used for assigning dendrite end points and dendrite surface rendering. To trace spines, the maximum spine length and minimum spine end diameter were set at $5 \mu \mathrm{m}$ and $0.215 \mu \mathrm{m}$, respectively, for fixed neuron experiments and $15 \mu \mathrm{m}$ and $0.3 \mu \mathrm{m}$, respectively, for live imaging experiments. Automatic thresholds were used for generating spine seed points and surface rendering. After generating the trace, a filter was applied to ensure all dendritic protrusions $\leq 5 \mu \mathrm{m}$ (or $15 \mu \mathrm{m}$ ) were assigned as spines; to do so, we created a filter that selected all dendritic segments with "Branch level" = 2 and "length" $\leq 5$ (or 15) 
and the selected segments were assigned as spines by choosing "Assign as spine" under the Edit tab. All of the geometric parameters and filters were set, or loaded from a previously analyzed image, at the start of the analysis session after which the software maintained these values. For each subsequent image processed, an ROI was selected, a dendrite starting point was assigned, and then the trace was built by clicking "Finish". To apply the filter, the Filter tab was opened (which automatically selected the appropriate segments), then by clicking on the Edit tab followed by "Assign as spine" the final 3D trace was generated. Filament statistics were exported into Excel (Microsoft), where they were compiled and graphed.

\section{Manual image analysis}

Manual analyses were performed in Imaris Surpass mode using the same dendritic ROIs as above. The dendrite length was measured using Measurement Points and each spine was marked using Spots (Imaris). Using Measurement Points, head width was measured at the maximum width of the spine tip, neck width was measured at the minimum point along the spine length, and spine length was measured from the dendrite shaft to the spine tip. Each ROI was processed in duplicate and the values were averaged.

\section{Spine classifications}

Spines were classified into groups termed stubby, mushroom, and thin. These groups were established as follows: stubby (length $\leq 1 \mu \mathrm{m}$ and neck width/head width $<1.5$ ), mushroom (neck width/head width $\geq 1.5$ and length $\leq 5 \mu \mathrm{m})$, and thin $(1<$ length $\leq 5 \mu \mathrm{m}$ and neck width/head width $<1.5)$ [36]. Classification for both manual and Filament Tracer, were computed in Excel using the following formulas:

$$
\begin{aligned}
\text { Stubby }: & =F(\operatorname{AND}(\text { length } \leq 5, \text { head } / \text { neck } \leq 1.5), 1,0) \\
\text { Mushroom }: & =\operatorname{IF}(\operatorname{AND}(\text { length } \leq 5, \text { head } / \text { neck } \geq 1.5), 1,0) \\
\text { Thin }: & =\operatorname{IF}(\operatorname{AND}(\text { length } \leq 5, \text { length }>1, \text { head } / \text { neck } \leq 1.5), 1,0)
\end{aligned}
$$

These logic statements return a value of 1 if true and 0 if false. The total number of spines in each class was tallied by summing the results of the logic statements. For live imaging experiments, a maximum length of 15 $\mu \mathrm{m}$ was used instead of $5 \mu \mathrm{m}$.

\section{Statistics}

Unless otherwise noted, statistics were completed using PASW Statistics 18 (SPSS, Inc). All datasets were analyzed for equal variance using Levene's test and normality using the Kolmogorov Smirnov test. Normally distributed datasets were compared using either Student's t-test or an ANOVA followed by post-hoc tests as noted in figure legends. Non-normal datasets were compared using the
Mann-Whitney U test or Kruskal Wallis test. Cumulative distributions were compared using the KolmogorovSmirnov test. Alpha was set at 0.05 for all comparisons. Power analysis was performed using G* Power 3.1.2 (University of Kiel, Germany) with $\beta=0.8$ and $\alpha=0.05$, and effect size and standard deviation were determined using pilot experiment results. The experimenter was blind to treatment and genotype during all image analysis.

\section{Additional material}

Additional file 1: Individual dendritic spines undergo remodeling that can be tracked across time. A 12 DIV hippocampal neuron expressing Lifeact-ruby was treated with BDNF $(100 \mathrm{ng} / \mathrm{ml})$ and image at 5 in intervals for $1 \mathrm{hr}$. In this representative neuron, a thin protrusion undergoes extensive remodeling (blue) and a newly formed spine emerges from the dendritic shaft (red) and morphs into a mushroomshaped spine.

Additional file 2: Stubby spines are highly stable following BDNF stimulation. A 12 DIV hippocampal neuron expressing Lifeact-ruby was treated with BDNF $(100 \mathrm{ng} / \mathrm{ml})$ and imaged at 5 min intervals for $1 \mathrm{hr}$. The stubby spine (center) remains stable for the duration of the experiment; whereas, neighboring thin protrusions can be seen extending and retracting.

Additional file 3: Thin protrusions with a high head/neck width ratio remain stable. A 12 DIV hippocampal neuron expressing Lifeactruby was treated with $100 \mathrm{ng} / \mathrm{ml}$ BDNF and imaged at 5 min intervals for $1 \mathrm{hr}$. The long protrusion with a defined head that is much wider than the neck (at right) remains stable throughout the experiment; whereas, neighboring protrusions, which do not exhibit increased width at the tip, undergo dynamic structural changes and/or are pruned.

\section{Acknowledgements}

The authors would like to thank Dr. Alexa Mattheyses for technical assistance and critically reading the manuscript and Andrew Swanson for technical assistance and helpful discussions. SAS was supported by predoctoral fellowships from the NIH (F31NS063668, T32GM0860512 and T32NS007480) and the Epilepsy Foundation and Lennox \& Lombroso Trust Fund. CG was supported by a Basic Science Grant from NFXF. This work was supported by $\mathrm{NIH} \mathrm{MH085617}$ and NARSAD Investigator Award to GJB, the Emory/Baylor Fragile X Center (P30HD024064) and the Neuronal Imaging Core of the Emory Neuroscience NINDS Core Facility (P30NS055077).

\section{Author details}

'Department of Cell Biology, Emory University, 615 Michael St. NE, Atlanta, GA 30322, USA. ²Department of Neurology, Emory University, 615 Michael St. NE, Atlanta, GA 30322, USA.

\section{Authors' contributions}

SAS contributed to the conception and design of the study, tested and validated the method, performed and analyzed the live imaging experiments, performed the statistical analyses, and drafted the manuscript. $X Y$ performed the mouse neuron culture, transfection, and imaging for the LY294002 experiments. CG participated in the design and analysis of the LY294002 experiments. GJB contributed to the conception, analysis, and coordination of the study, and edited the manuscript. All authors have read and approved the final manuscript.

\section{Competing interests}

CG and GJB declare that they are inventors on patent application PCT/ US2010/055387.

Received: 29 August 2011 Accepted: 7 October 2011

Published: 7 October 2011 


\section{References}

1. Edwards FA: Anatomy and electrophysiology of fast central synapses lead to a structural model for long-term potentiation. Physiol Rev 1995, 75:759-787.

2. Holtmaat A, Svoboda K: Experience-dependent structural synaptic plasticity in the mammalian brain. Nat Rev Neurosci 2009, 10:647-658.

3. Kasai H, Fukuda M, Watanabe S, Hayashi-Takagi A, Noguchi J: Structural dynamics of dendritic spines in memory and cognition. Trends Neurosci 2010, 33:121-129.

4. Alvarez VA, Sabatini BL: Anatomical and physiological plasticity of dendritic spines. Annu Rev Neurosci 2007, 30:79-97.

5. Penzes $P$, Cahill ME, Jones KA, Vanleeuwen JE, Woolfrey KM: Dendritic spine pathology in neuropsychiatric disorders. Nat Neurosci 2011, 14:285-293.

6. Lee KJ, Kim H, Rhyu IJ: The roles of dendritic spine shapes in Purkinje cells. Cerebellum 2005, 4:97-104.

7. Glantz LA, Lewis DA: Dendritic spine density in schizophrenia and depression. Arch Gen Psychiatry 2001, 58:203.

8. Hayashi Y, Majewska AK: Dendritic spine geometry: functional implication and regulation. Neuron 2005, 46:529-532.

9. Bourne $\mathrm{JN}$, Harris KM: Coordination of size and number of excitatory and inhibitory synapses results in a balanced structural plasticity along mature hippocampal CA1 dendrites during LTP. Hippocampus 2010.

10. Bourne JN, Harris KM: Balancing structure and function at hippocampal dendritic spines. Annu Rev Neurosci 2008, 31:47-67.

11. Svoboda K: The past, present, and future of single neuron reconstruction. Neuroinformatics 2011, 9:97-98.

12. Rodriguez A, Ehlenberger DB, Dickstein DL, Hof PR, Wearne SL: Automated three-dimensional detection and shape classification of dendritic spines from fluorescence microscopy images. PLoS One 2008, 3:e1997.

13. Rodriguez A, Ehlenberger DB, Hof PR, Wearne SL: Rayburst sampling, an algorithm for automated three-dimensional shape analysis from laser scanning microscopy images. Nature protocols 2006, 1:2152-2161.

14. Wearne SL, Rodriguez A, Ehlenberger DB, Rocher AB, Henderson SC, Hof PR: New techniques for imaging, digitization and analysis of threedimensional neural morphology on multiple scales. Neuroscience 2005, 136:661-680

15. Janoos F, Mosaliganti K, Xu X, Machiraju R, Huang K, Wong ST: Robust 3D reconstruction and identification of dendritic spines from optical microscopy imaging. Med Image Anal 2009, 13:167-179.

16. Zhang Y, Chen K, Baron M, Teylan MA, Kim Y, Song Z, Greengard P, Wong ST: A neurocomputational method for fully automated 3D dendritic spine detection and segmentation of medium-sized spiny neurons. Neuroimage 2010, 50:1472-1484.

17. Zhang Y, Zhou X, Witt RM, Sabatini BL, Adjeroh D, Wong ST: Dendritic spine detection using curvilinear structure detector and LDA classifier. Neuroimage 2007, 36:346-360.

18. Mukai H, Hatanaka Y, Mitsuhashi K, Hojo Y, Komatsuzaki Y, Sato R, Murakami G, Kimoto T, Kawato S: Automated Analysis of Spines from Confocal Laser Microscopy Images: Application to the Discrimination of Androgen and Estrogen Effects on Spinogenesis. Cerebral cortex 2011.

19. Bloss EB, Janssen WG, Ohm DT, Yuk FJ, Wadsworth S, Saardi KM, McEwen BS, Morrison JH: Evidence for reduced experience-dependent dendritic spine plasticity in the aging prefrontal cortex. J Neurosci 2011, 31:7831-7839.

20. Scotto-Lomassese S, Nissant A, Mota T, Neant-Fery M, Oostra BA, Greer CA Lledo PM, Trembleau A, Caille I: Fragile $X$ mental retardation protein regulates new neuron differentiation in the adult olfactory bulb. $J$ Neurosci 2011, 31:2205-2215.

21. Stevens JK, Trogadis J: Reconstructive three-dimensional electron microscopy. A routine biologic tool. Anal Quant Cytol Histol 1986, 8:102-107

22. Shen $\mathrm{H}$, Sesack SR, Toda S, Kalivas PW: Automated quantification of dendritic spine density and spine head diameter in medium spiny neurons of the nucleus accumbens. Brain Struct Funct 2008, 213:149-157.

23. Staffend NA, Loftus CM, Meisel RL: Estradiol reduces dendritic spine density in the ventral striatum of female Syrian hamsters. Brain Struct Funct 2011, 215:187-194

24. Gross C, Nakamoto M, Yao X, Chan CB, Yim SY, Ye K, Warren ST, Bassell GJ: Excess phosphoinositide 3-kinase subunit synthesis and activity as a novel therapeutic target in fragile $X$ syndrome. J Neurosci 2010, 30:10624-10638

25. Tanaka J, Horiike Y, Matsuzaki M, Miyazaki T, Ellis-Davies GC, Kasai H: Protein synthesis and neurotrophin-dependent structural plasticity of single dendritic spines. Science 2008, 319:1683-1687.

26. Bassell GJ, Warren ST: Fragile $X$ syndrome: loss of local mRNA regulation alters synaptic development and function. Neuron 2008, 60:201-214.

27. Riedl J, Crevenna AH, Kessenbrock K, Yu JH, Neukirchen D, Bista M, Bradke F, Jenne D, Holak TA, Werb Z, et al: Lifeact: a versatile marker to visualize F-actin. Nat Methods 2008, 5:605-607.

28. Riedl J, Flynn KC, Raducanu A, Gartner F, Beck G, Bosl M, Bradke F, Massberg S, Aszodi A, Sixt M, Wedlich-Soldner R: Lifeact mice for studying F-actin dynamics. Nat Methods 2010, 7:168-169.

29. Papa M, Bundman MC, Greenberger V, Segal M: Morphological analysis of dendritic spine development in primary cultures of hippocampal neurons. J Neurosci 1995, 15:1-11.

30. Ludbrook J: Linear regression analysis for comparing two measurers or methods of measurement: but which regression? Clin Exp Pharmacol Physiol 2010, 37:692-699.

31. Harris KM, Stevens JK: Dendritic spines of CA 1 pyramidal cells in the rat hippocampus: serial electron microscopy with reference to their biophysical characteristics. J Neurosci 1989, 9:2982-2997.

32. Kasai H, Matsuzaki M, Noguchi J, Yasumatsu N, Nakahara H: Structurestability-function relationships of dendritic spines. Trends Neurosci 2003, 26:360-368.

33. Noguchi J, Matsuzaki M, Ellis-Davies GC, Kasai H: Spine-neck geometry determines NMDA receptor-dependent $\mathrm{Ca} 2+$ signaling in dendrites. Neuron 2005, 46:609-622.

34. Korkotian E, Holcman D, Segal M: Dynamic regulation of spine-dendrite coupling in cultured hippocampal neurons. Eur J Neurosci 2004, 20:2649-2663.

35. Biess A, Korkotian E, Holcman D: Diffusion in a dendritic spine: the role of geometry. Phys Rev E Stat Nonlin Soft Matter Phys 2007, 76:021922.

36. Harris KM, Jensen FE, Tsao B: Three-dimensional structure of dendritic spines and synapses in rat hippocampus (CA1) at postnatal day 15 and adult ages: implications for the maturation of synaptic physiology and long-term potentiation. J Neurosci 1992, 12:2685-2705

37. Peters A, Kaiserman-Abramof IR: The small pyramidal neuron of the rat cerebral cortex. The perikaryon, dendrites and spines. Am J Anat 1970, 127:321-355

38. Fan J, Zhou X, Dy JG, Zhang Y, Wong ST: An automated pipeline for dendrite spine detection and tracking of 3D optical microscopy neuron images of in vivo mouse models. Neuroinformatics 2009, 7:113-130.

39. Koh IY, Lindquist WB, Zito K, Nimchinsky EA, Svoboda K: An image analysis algorithm for dendritic spines. Neural Comput 2002, 14:1283-1310.

40. Matsuzaki M, Honkura N, Ellis-Davies GC, Kasai H: Structural basis of longterm potentiation in single dendritic spines. Nature 2004, 429:761-766.

41. Zhou Q, Homma KJ, Poo MM: Shrinkage of dendritic spines associated with long-term depression of hippocampal synapses. Neuron 2004, 44:749-757.

42. Yoshihara $Y$, De Roo M, Muller D: Dendritic spine formation and stabilization. Current opinion in neurobiology 2009, 19:146-153.

43. Heiman MG, Shaham S: Twigs into branches: how a filopodium becomes a dendrite. Current opinion in neurobiology 2010, 20:86-91.

44. Gottmann K, Mittmann T, Lessmann V: BDNF signaling in the formation, maturation and plasticity of glutamatergic and GABAergic synapses. Exp Brain Res 2009, 199:203-234.

45. Chapleau CA, Larimore JL, Theibert A, Pozzo-Miller L: Modulation of dendritic spine development and plasticity by BDNF and vesicular trafficking: fundamental roles in neurodevelopmental disorders associated with mental retardation and autism. J Neurodev Disord 2009, 1:185-196

46. Bourne J, Harris KM: Do thin spines learn to be mushroom spines that remember? Curr Opin Neurobiol 2007, 17:381-386.

47. Fiala JC, Feinberg M, Popov V, Harris KM: Synaptogenesis via dendritic filopodia in developing hippocampal area CA1. J Neurosci 1998, 18:8900-8911.

48. Matsuo N, Reijmers L, Mayford M: Spine-type-specific recruitment of newly synthesized AMPA receptors with learning. Science 2008, 319:1104-1107. 
49. Ashby MC, Maier SR, Nishimune A, Henley JM: Lateral diffusion drives constitutive exchange of AMPA receptors at dendritic spines and is regulated by spine morphology. J Neurosci 2006, 26:7046-7055.

50. Harris KM: Structure, development, and plasticity of dendritic spines. Curr Opin Neurobiol 1999, 9:343-348.

51. Richardson RJ, Blundon JA, Bayazitov IT, Zakharenko SS: Connectivity patterns revealed by mapping of active inputs on dendrites of thalamorecipient neurons in the auditory cortex. J Neurosci 2009, 29:6406-6417.

52. Holtmaat AJ, Trachtenberg JT, Wilbrecht L, Shepherd GM, Zhang X, Knott GW, Svoboda K: Transient and persistent dendritic spines in the neocortex in vivo. Neuron 2005, 45:279-291

53. Zuo Y, Lin A, Chang P, Gan WB: Development of long-term dendritic spine stability in diverse regions of cerebral cortex. Neuron 2005, 46:181-189.

54. Yuste R, Bonhoeffer T: Genesis of dendritic spines: insights from ultrastructural and imaging studies. Nature reviews Neuroscience 2004, 5:24-34.

55. Papa M, Segal M: Morphological plasticity in dendritic spines of cultured hippocampal neurons. Neuroscience 1996, 71:1005-1011.

56. Ziv NE, Smith SJ: Evidence for a role of dendritic filopodia in synaptogenesis and spine formation. Neuron 1996, 17:91-102.

57. Marrs GS, Green SH, Dailey ME: Rapid formation and remodeling of postsynaptic densities in developing dendrites. Nat Neurosci 2001, 4:1006-1013.

58. Okabe S, Miwa A, Okado H: Spine formation and correlated assembly of presynaptic and postsynaptic molecules. J Neurosci 2001, 21:6105-6114.

59. Knott GW, Holtmaat A, Wilbrecht L, Welker E, Svoboda K: Spine growth precedes synapse formation in the adult neocortex in vivo. Nat Neurosci 2006, 9:1117-1124.

60. Nagerl UV, Kostinger G, Anderson JC, Martin KA, Bonhoeffer T: Protracted synaptogenesis after activity-dependent spinogenesis in hippocampal neurons. J Neurosci 2007, 27:8149-8156.

61. Kwon HB, Sabatini BL: Glutamate induces de novo growth of functional spines in developing cortex. Nature 2011, 474:100-104.

62. Konur S, Yuste R: Imaging the motility of dendritic protrusions and axon terminals: roles in axon sampling and synaptic competition. Molecular and cellular neurosciences 2004, 27:427-440.

63. Lohmann C, Finski A, Bonhoeffer T: Local calcium transients regulate the spontaneous motility of dendritic filopodia. Nat Neurosci 2005, 8:305-312.

64. Grunditz A, Holbro N, Tian L, Zuo Y, Oertner TG: Spine neck plasticity controls postsynaptic calcium signals through electrical compartmentalization. J Neurosci 2008, 28:13457-13466.

65. Irwin SA, Patel B, Idupulapati M, Harris JB, Crisostomo RA, Larsen BP, Kooy F, Willems PJ, Cras P, Kozlowski PB, et al: Abnormal dendritic spine characteristics in the temporal and visual cortices of patients with fragile-X syndrome: a quantitative examination. Am J Med Genet 2001 98:161-167.

66. Irwin SA, Idupulapati M, Gilbert ME, Harris JB, Chakravarti AB, Rogers EJ, Crisostomo RA, Larsen BP, Mehta A, Alcantara CJ, et al: Dendritic spine and dendritic field characteristics of layer $\mathrm{V}$ pyramidal neurons in the visual cortex of fragile-X knockout mice. Am J Med Genet 2002, 111:140-146.

67. Antar LN, Li C, Zhang H, Carroll RC, Bassell GJ: Local functions for FMRP in axon growth cone motility and activity-dependent regulation of filopodia and spine synapses. Molecular and cellular neurosciences 2006, 32:37-48.

68. Cruz-Martin A, Crespo M, Portera-Cailliau C: Delayed stabilization of dendritic spines in fragile X mice. J Neurosci 2010, 30:7793-7803.

69. Bilousova TV, Dansie L, Ngo M, Aye J, Charles JR, Ethell DW, Ethell IM: Minocycline promotes dendritic spine maturation and improves behavioural performance in the fragile X mouse model. J Med Genet 2009, 46:94-102.

70. Galvez R, Greenough WT: Sequence of abnormal dendritic spine development in primary somatosensory cortex of a mouse model of the fragile $X$ mental retardation syndrome. American journal of medical genetics Part A 2005, 135:155-160.

71. de Vrij FM, Levenga J, van der Linde HC, Koekkoek SK, De Zeeuw Cl, Nelson DL, Oostra BA, Willemsen R: Rescue of behavioral phenotype and neuronal protrusion morphology in Fmr1 KO mice. Neurobiology of disease 2008, 31:127-132.
72. Gross C, Berry-Kravis EM, Bassell GJ: Therapeutic Strategies in Fragile X Syndrome: Dysregulated mGluR Signaling and Beyond. Neuropsychopharmacology 2011.

73. Portera-Cailliau C: Which Comes First in Fragile X Syndrome, Dendritic Spine Dysgenesis or Defects in Circuit Plasticity? Neuroscientist 2011.

74. Rocher AB, Kinson MS, Luebke Jl: Significant structural but not physiological changes in cortical neurons of 12-month-old Tg2576 mice. Neurobiology of disease 2008, 32:309-318.

75. Tiruchinapalli DM, Oleynikov Y, Kelic S, Shenoy SM, Hartley A, Stanton PK, Singer RH, Bassell GJ: Activity-dependent trafficking and dynamic localization of zipcode binding protein 1 and beta-actin mRNA in dendrites and spines of hippocampal neurons. J Neurosci 2003, 23:3251-3261.

76. Chen Y, Stevens B, Chang J, Milbrandt J, Barres BA, Hell JW: NS21: redefined and modified supplement B27 for neuronal cultures. J NeurosCi Methods 2008, 171:239-247.

77. Sorra KE, Harris KM: Overview on the structure, composition, function, development, and plasticity of hippocampal dendritic spines. Hippocampus 2000, 10:501-511.

doi:10.1186/1756-6606-4-38

Cite this article as: Swanger et al:: Automated 4D analysis of dendritic spine morphology: applications to stimulus-induced spine remodeling and pharmacological rescue in a disease model. Molecular Brain 2011 $4: 38$.

\section{Submit your next manuscript to BioMed Central and take full advantage of:}

- Convenient online submission

- Thorough peer review

- No space constraints or color figure charges

- Immediate publication on acceptance

- Inclusion in PubMed, CAS, Scopus and Google Scholar

- Research which is freely available for redistribution 